UNANGENEHME MITREISENDE

\title{
Immer wieder Norovirus-Infektionen in einer Boeing 777
}

- Norovirus-Infektionen sind hoch ansteckend: Das bestätigt eine auffällige Serie von Brechdurchfällen bei Flugbegleitern von Air New Zealand. Nachforschungen ergaben, dass alle Erkrankten in demselben Flugzeug gearbeitet hatten.

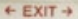

$\longrightarrow$

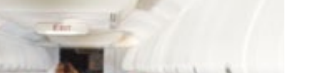

Von insgesamt 77 Flugbegleitern, die sich zwischen dem 13. und 18. Oktober 2009 an Bord der Boeing 777 aufgehalten hatten, entwickelten 29 nach ihrem Flug Diarrhö und/oder Erbrechen. Ein Steward berichtete, dass sich am 13. Oktober in der Economy Class ein Passagier übergeben und dabei den Teppich verschmutzt hatte.

Mehrere Beobachtungen sprechen dafür, dass sich das Servicepersonal in diesem Flugzeug mit Noroviren infiziert hatte. Die Infektionsrate lag bei 43\%. Das häufigste Symptom war Diarrhö (89\%), gefolgt von Übelkeit (82\%), Bauchschmerzen (70\%), Fieber (67\%) und Erbrechen (56\%). Inwieweit sich Fluggäste mit dem Norovirus angesteckt hatten, wurde aus logistischen Gründen nicht erhoben.

Die Krankheitsserie konnte gestoppt werden, indem das Innere der Boeing komplett dekontaminiert wurde. Dazu wurden unter anderem alle Oberflächen mit 0,2\%igem Parachlorometaxylenol desinfiziert.

$B S=$

Auch die Viren fliegen mit.
UNAUSGESCHLAFEN IM OP

\section{Macht Kaffee müde Chirurgen munter?}

- Wenn Chirurgen unter Schlafmangel leiden, wird die Fehlerquote und damit die Patientensicherheit auch durch Einnahme von Koffein oder Taurin nicht verbessert.

Die Leistung von 18 Medizinstudenten, die ein Training an einem Laparoskopie-Simulator erhalten hatten, wurde zunächst in ausgeruhtem Zustand dokumentiert. In drei weiteren Runden durften sie 24 Stunden lang nicht schlafen und erhielten dann eine Stunde vor den Tests Tabletten mit Vitamin C und Kalzium (= Placebo), mit Koffein oder mit Koffein plus Taurin.

Nach Schlafentzug (unter Placebo) brauchten die Probanden länger, waren weniger ökonomisch in ihren Bewegungen und machten mehr Fehler als nach ausreichend Schlaf. Unter Koffein agierten sie zwar schneller und ökonomischer, aber sie machten nicht weniger Fehler. Mit Koffein plus Taurin war das Ergebnis ähnlich. BS • BrJ Surg online August 2011

\section{Risikogruppen konsequent testen}

— Für ein risikogruppenadaptiertes Laborscreening auf chronische Hepatitis B plädiert die Deutsche Leberhilfe. Auf welchen Wegen sich dieses Ziel erreichen ließe, erläuterte Prof. Claus Niederau, Oberhausen.

Getestet gehören laut Niederau zunächst einmal Menschen, bei denen erhöhte Transaminasen auffallen - unabhängig davon, wie stark die Messergebnisse von der Norm abweichen. „Es darf nicht sein, dass Menschen jahrelang mit solchen Werten herumlaufen, ohne dass jemals eine HepatitisSerologie angefordert wird", unterstrich Niederau.

Das typische Symptom Müdigkeit helfe in der Praxis nicht weiter; und bis zum Ikterus dürfe es eigentlich gar nicht kommen. Es sei aus diesem
Grund sinnvoll, Menschen, die ein gewisses Risiko tragen, einen Test auf Hepatitis $B$ anzubieten.

Zu den Risikogruppen, die konsequent auf chronische Virushepatitis untersucht werden sollten, zählt Niederau u. a. Menschen mit häufig wechselnden Sexualpartnern, Drogenabhängige und Angehörige medizinischer Berufe. Zu den Gefährdeten seien darüber hinaus Migranten erster Generation aus der Türkei, Asien, den Gebieten der ehemaligen Sowjetunion, Afrika und den Mittelmeerländern zu rechnen. Von den schätzungsweise 500000 Menschen in Deutschland, die eine chronische Hepatitis $B$ aufweisen, gehörten rund zwei Drittel zu dieser Gruppe.

Viele Patienten mit Hepatitis $B$ hätten normale Leberwerte. „Deswegen macht es bei Risikogruppen Sinn, auch ohne erhöhte

\section{Deutsche Leberhilfe e.V.}

Transaminasen zu testen", sagte Niederau. Dies gelte besonders für $\mathrm{Mi}$ granten. „Die haben sich fast alle in ihrem Herkunftsland infiziert."

Geholfen wird mit dem Screening auf Hepatitis B nach Ansicht des Leberexperten zunächst den Betroffenen. Chronische Hepatitis B sei zwar meist nicht heilbar, aber gut zu therapieren. Von den Tests profitieren ferner die Mitmenschen: „Denn anders als bei der chronischen Hepatitis C, bei der die Gefahr einer Übertragung von Mensch zu Mensch gering ist, korreliert das Übertragungsrisiko bei der chronischen Hepatitis B deutlich mit der Virusmenge."

RB =

Weitere Informationen auf den Seiten der Deutschen Leberhilfe im Internet unter http://www.leberhilfe. org/ 